\title{
LA INVESTIGACIÓN EN GEOMORFOLOGÍA PERIGLACIAR EN ESPAÑA Y PORTUGAL. EVOLUCIÓN RECIENTE Y ESTUDIOS ACTUALES
}

\author{
Antonio Gómez OrTiz ${ }^{1}$ \\ Gonçalo T. VieIRA ${ }^{2}$
}

\begin{abstract}
Resumen - Se hace una revisión de la producción científica reciente por parte de autores españoles y portugueses en temas de geomorfología periglaciar a partir de las publicaciones del grupo de trabajo IPA-España. Su valoración crítica permite explicar la evolución de este conocimiento disciplinar (ideas, conceptos, métodos, ámbitos y temas de estudio y grupos de investigación) en el contexto del progreso que la Geomorfología ha venido experimentando en ambos países. Acontecimientos significativos en todo ello fueron la creación de la Sociedad Española de Geomorfología (SEG), en 1987; el Grupo Español IPA-España, en 1994, y el Grupo Portugués IPA-Portugal, en 2005, ambos inmersos en la International Permafrost Association.
\end{abstract}

Palabras clave: Geomorfología periglaciar, estudios recientes, España, Portugal.

Resumo - A INVESTIGAÇÃo EM GEOMORFOLOGIA PERIGLACIÁRIA EM ESPANHA E Portugal. Evolução ReCENTE E EStudos ACtuAis. Apresenta-se uma síntese dos trabalhos recentes de autores espanhóis e portugueses no âmbito da geomorfologia periglaciária. O levantamento efectuado baseia-se na análise crítica (ideias, conceitos, métodos, áreas e temas de estudo e, grupos de trabalho) das publicações do grupo de trabalho IPA-Espanha. Esta é enquadrada no contexto dos progressos que os estudos de Geomorfologia vêm experimentando em ambos os países, de modo a explicar a evolução científica recente. Os principais marcos na evolução dos estudos de periglaciarismo na Península Ibérica são a criação da Sociedade Espanhola de Geomorfologia em 1987, do grupo espanhol da International Permafrost Association em 1994, e do grupo português da mesma associação em 2005.

Palavras-chave: Geomorfologia periglaciária, investigação recente, Espanha, Portugal.

Abstract - Research on Periglacial geomorphology in Spain and Portugal. TRENDS AND CONTEMPORARY STUDIES. An analysis of the scientific literature on

1 Área de Geografía física y Servei de Paisatge de la Universidad de Barcelona. E-mail: gomez@ub.edu

2 Investigador do Centro de Estudos Geográficos e Professor Auxiliar da Faculdade de Letras da Universidade de Lisboa. E-mail: gtvieira@ceg.ul.pt 
periglacial geomorphology produced by Spanish and Portuguese researchers is presented. The paper is based in the proceedings of the meetings of the IPA-Spain group. The critical evaluation of these publications allows explaining the evolution of Iberian periglacial geomorphology (i.e. ideas, concepts, methods, regions of study, themes and research groups) in the context of the evolution that Geomorphology as a science is facing in both countries. Given this framework, the more significant events that occurred were the founding of the Spanish Society of Geomorphology (SEG) in 1987, of the Spanish Group of the International Permafrost Association (IPA-Spain) in 1994, and of the Portuguese Group (IPA-Portugal) in 2005.

Key words: Periglacial geomorphology, recent research, Spain, Portugal.

Résumé - LA RECHERCHE EN GÉOMORPHOLOGIE DU PÉRIGLACIAIRE EN ESPAGNE ET AU Portugal. Évolution RÉCENTE ET ÉTUdes EN COURS. On présente la production scientifique récente des auteurs espagnols et portugais dans le domaine de la Géomorphologie du périglaciaire, en se basant sur les comptes-rendus des réunions du Groupe de Travail IPA-Espagne. Leur présentation critique permet de montrer l'évolution des idées, des concepts et des méthodes, ainsi que des thèmes et lieux de recherche qui sont pratiqués par les divers groupes constitués. Les événements les plus significatifs ont été la création de la Sociedad Española de Geomorfologia en 1897, du Grupo Español IPA-España en 1994 et du Grupo Português IPA-Portugal en 2005, ces deux derniers faisant partie de la International Permafrost Association.

Mots-clés: Géomorphologie périglaciaire, recherche, Espagne, Portugal.

\section{ANTECEDENTES}

El interés por el estudio de las formas de relieve asociadas a procesos periglaciares por la comunidad científica ibérica es relativamente reciente. Esta labor podría instalarse a partir de la primera mitad del siglo XX, probablemente como respuesta a la celebración del V Congreso Internacional de INQUA (Madrid-Barcelona, 1957), donde se dio cuenta de la existencia de formas de modelado de origen frío no glaciar en diferentes lugares de la Península Ibérica. La fecha es significativa si tenemos en consideración que la Unión Geográfica Internacional, en su XVI Congreso, celebrado en Lisboa en 1949, creó la Comisión sobre periglaciarismo (Pissart, 1999). Sin embargo, hay que reseñar que mucho antes ya habían sido señalados registros periglaciares. Por ejemplo, en Sierra Nevada (Dresch, 1937), Pirineo central (García Sainz, 1935; Barrère, 1952), oeste de Lisboa (Guilcher, 1949), Macizo Calcário Estremenho (Martins, 1949). $\mathrm{E}$, incluso, por estos mismos años se comenzó a reflexionar sobre el tema, como así lo hizo Floristán (1953), en su librito El modelado periglaciar donde resalta el significado de la morfodinámica de procesos fríos en la definición de muchos de los relieves de latitudes medias bajo condiciones no glaciares. El progreso del conocimiento periglaciar hasta entrada la década de los años 70 del siglo XX fue lento aunque progresivo y se resolvió, sobre todo, en la catalogación, descripción y cartografia de modelados (Peña Monné, 1998). Los autores de estos estudios fueron, en su mayoría, extranjeros y focalizaron esfuerzos en montaña y sectores aledaños (Carvalho, 1955, 1964; Hamelin, 1958; Hempel, 1960; Messerli, 1965; Tricart, 1966; Höllermann, 1967; Brochu, 1969; 
Soutadé y Baudière, 1970; Daveau, 1973; Brosche, 1978; etc). El verdadero aporte científico autóctono se operó en el tránsito de la siguiente década, la de los 80 , y vino influido, muy particularmente en España, por la escuela francesa de Estrasburgo, pues principios, ideas, métodos de trabajo y técnicas cartográficas fueron introducidas a través de Tricart $(1962,1967$, etc.). En Portugal la influencia francesa también fue notoria a través de los trabajos de Carvalho $(1955,1964)$ y Daveau $(1973,1978)$.

\section{EL PROGRESO OPERADO HASTA LA DÉCADA DE LOS AÑOS 80 DEL SIGLO XX}

Fue, por tanto, a partir de finales de la década de los 70 cuando empezó a irrumpir con paso firme en la literatura geomorfológica española y portuguesa lo relativo a las formas de modelado periglaciar centrando atención, sobre todo, en ámbitos montañosos de la Península Ibérica, en especial en Pirineo, Cordillera Ibérica, sierras de Guadarrama-Gredos, serra da Estrela y algunos enclaves de la cuenca del Duero y Tajo, preocupando, particularmente, la evolución de laderas. El interés geomorfológico por las manifestaciones periglaciares pronto se generalizó entre la comunidad de geomorfólogos (geógrafos y geólogos) y el dato más objetivo durante estos tiempos fueron las publicaciones (García Ruiz, 1999) y la irrupción de esta temática en los certámenes científicos sobre cuestiones cuaternarias y geográficas. Al respecto, resulta ya significativa la presencia de comunicaciones de carácter periglaciar en la II. ${ }^{a}$ Reunión del Grupo Español para el Estudio del Cuaternario (Jaca, 1975) y en el V Coloquio de Geografía (Granada, 1976). El comentario que puede hacerse de estos trabajos, en número de 8 y 10, respectivamente, es la preocupación generalizada que suscitan las formas frías no glaciares en la definición del modelado de laderas en montañas de altitud modesta y en algunas, incluso, cercanas a la línea de costa. Esta constatación significó entreabrir el debate, aún vigente, acerca de la magnitud de las condiciones frías pleistocenas en la Península Ibérica, de los procesos desencadenantes y de su resultado en la configuración de los actuales relieves, cuestión, por cierto, ya planteada por Rosselló (1970) para el ámbito mediterráneo y remarcada, de nuevo por él mismo, en la Ponencia que abrió las sesiones del referido Coloquio de Geografía (Rosselló, 1977).

De esta época son también las primeras tesis doctorales sobre procesos fríos en las que sin olvidar la acción glaciar lo relativo a morfodinámica periglaciar ocupó lugar destacado, en ocasiones predominante o, incluso, exclusivo. En tal sentido, merece destacarse la atención que suscitó el Pirineo donde se describieron y explicaron morfologías heredadas y actuales (Serrat, 1977; Gómez Ortiz, 1980; Peña Monné, 1980; etc.) y donde, además, se interpretaron los procesos fríos actuales como agentes muy eficaces y significativos en la definición del paisaje de los niveles supraforestales (Soutadé, 1978). De estos mismos años también fue el conocimiento de modelados periglaciares en las sierras de Peneda y Gerês (Minho-Portugal) (Coudé-Gaussen, 1979).

En semejante línea investigadora el despliegue de artículos publicados en revistas y contribuciones a reuniones científicas por parte de los investigadores españoles y portugueses se incrementó notablemente, ocupando lugar relevante el descubrimiento de formas heredadas y su cartografía precisa tanto en áreas glaciadas como en aquellas otras no glaciadas. La producción científica, abundante en número y temas tratados, incluyó los sistemas montañosos más importantes de la Península Ibérica, aunque ocupando lugar preferente el Sistema Central (Martínez de Pisón y Muñoz Jiménez, 
1972; Sanz Herraiz, 1977; Bullón Mata, 1977; Pedraza Gilsanz, 1978) y el Pirineo (Gómez Ortiz y Serrat, 1977), en el que por primera vez se describen trabajos experimentales de campo en lóbulos de gelifluxión (Creus y García Ruiz, 1977). Destaca también la atención que empezaron a tener aquellas montañas con ligero rastro glaciar o sin él. De éstas caben destacarse el Prepirineo leridano (Solé Sugrañes, 1973), Montseny (Llobet, 1975/76), Moncayo (Pellicer Corellano, 1980), Javalambre (Gutiérrez y Peña Monné, 1975), Alto Maestrazgo (Ginés y Mateu, 1977), Sierra Mágina (García Rossell y Pezzi, 1977), etc. Igualmente sobresale el interés que despertaron las islas Baleares y Canarias describiéndose derrubios periglaciares en laderas de la serra de la Tramontana (Rosselló, 1977) y figuras geométricas actuales en las Cañadas del Teide (Morales et al., 1977). Asimismo mereció atención la Meseta española, pues del valle del Tajuña se analizaron derrubios ordenados (grèze litée) (Asensio Amor y González Martín, 1974), que también se señalaron para el SO de Galicia (Pérez Alberti, 1979). Y por lo que respecta a Portugal destacan los trabajos de Daveau (1973), sobre depósitos periglaciares heredados en las sierras de la Estrela y Lousã (Sistema Central) y Macizo Calcário Estremenho y margen litoral (norte de Lisboa); Rebelo (1975), que se ocupó de las crestas cuarcíticas de Valongo y depósitos asociados; Coudé-Gaussen (1979), acerca de la morfogénesis de origen frío en las sierras de Gerês y Peneda. Igualmente resalta por su novedad las reflexiones que Daveau (1978) hace del significado del frío actual en la morfodinámica del modelado de las montañas portuguesas.

Los resultados logrados hasta finales de la década de los 70 podrían resumirse en las siguientes ideas:

a) Desarrollo generalizado de los procesos fríos no glaciares en el conjunto de la Península Ibérica a lo largo del Pleistoceno;

b) Reparto espacial de formas de modelado periglaciar en montañas con desarrollo de glaciares cuaternarios o sin ellos y su reflejo en el margen litoral cántabro y portugués hasta la latitud de Lisboa;

c) Desarrollo temporal de procesos y formas periglaciares coetáneos y posteriores al glaciarismo, según tipo de montaña y cotas;

d) Interés preferente por el modelado de laderas (derrubios tipo grèze litée y groize);

e) Constatación de procesos periglaciares actuales en Pirineo central y Cañadas del Teide y

f) Preocupación dominante por la descripción de las formas y su cartografía.

\section{INVESTIGACIONES RECIENTES}

Las últimas décadas del siglo XX y los primeros años del XXI, desde 1980, el progreso operado en investigación periglaciar por parte de la comunidad española y portuguesa ha resultado muy notable y ha tenido un desarrollo paralelo a los avances que se han venido registrando en la geomorfología en general (Vieira y Cordeiro, 1998; Ferreira et al., 2000; Gómez Ortiz et al., 2001). El conocimiento de la morfogénesis fría y el número de áreas investigadas ha venido a confirmar la gran extensión que ocupan las formas de modelado frío no glaciar en el conjunto de la Península Ibérica así como la multivariedad de sus procesos desencadenantes. Asimismo se ha puesto de relieve la existencia de un piso periglaciar activo en determinadas unidades geográficas de algunas cordilleras así como la existencia de bolsas de permafrost alpino y funcionalidad de glaciares rocosos. 
El avance operado, siempre avalado por la asunción de nuevos conceptos e ideas, métodos y técnicas por parte de los grupos de investigación implicados, debió resultar de una serie de datos coincidentes, probablemente los mismos que caracterizan a la geomorfología:

a) el contacto con el exterior a través de la participación en programas de actuación internacional y eventos científicos;

b) la mayor comunicación entre disciplinas y entre colectivos con líneas de investigación afines y

c) la implantación de programas $\mathrm{I}+\mathrm{D}$ de alcance europeo, estatal y autonómico.

\section{La labor de la Sociedad Española de Geomorfología (SEG)}

El interés generalizado que despertaron los temas periglaciares a partir de la década de los 80, particularmente en España, vino favorecido, igualmente, por la Sociedad Española de Geomorfología (SEG), creada en 1987. Su labor en favor de la geomorfología española ha venido siendo valiosa, al tiempo que reconocida internacionalmente su trayectoria. La evidencia más relevante de estos hechos ha sido la realización, en Zaragoza, en septiembre de 2005, de la Sixth International Conference on Geomorphology. De las actividades de la SEG se destacan dos. La organización de sus reuniones nacionales y la publicación de sus actas (con frecuencia bianual desde 1990) (tabla 1) y la edición de la revista "Cuaternario y Geomorfología" (que edita conjuntamente con AEQUA).

Pero, probablemente, el acontecimiento más relevante durante sus primeros años fue la celebración de la primera reunión monográfica sobre periglaciarismo, que, en colaboración con el Servei de Paisatge de la Universidad de Barcelona y la Universidad de Granada, tuvo lugar en Granada, en 1993, donde se puso al día la situación de los estudios periglaciares en la Península Ibérica, Canarias y Baleares. La reunión, de la que se editó el monográfico pertinente, sin duda, marcó un hito muy significativo pues logró reunir por primera vez a los principales grupos de trabajo que venían ocupándose del tema, tanto españoles como portugueses.

\section{La Internacional Permafrost Association. Elemento catalizador de los estudios recientes sobre periglaciarismo en la Península Ibérica}

Otro de los acontecimientos decisivos que afianzó el progreso del estudio de la morfodinámica periglaciar fue la constitución, en 1994, del Grupo Español de la International Permafrost Association (IPA-España), cuyo cometido específico es el fomento de la investigación relacionada con los procesos fríos, particularmente de naturaleza periglaciar. El colectivo, que desde sus primeros años incluyó a investigadores portugueses, puede considerarse como uno de los más dinámicos y novedosos en el panorama de la geomorfología ibérica. Su perfil disciplinar viene aglutinando a diferentes especialistas instalados en distintas áreas de conocimiento, lo que ha llevado a interpretar el origen, desarrollo y resultados de los procesos fríos desde ópticas pluridisciplinares y complementarias. Esta perspectiva viene a mostrar no sólo la consolidación de la disciplina sino también la madurez y rigor de los planteamientos y resultados. En tal sentido, cobra especial relevancia el hecho de que cada vez más los objetivos de las investigaciones tienden a preocuparse por cuestiones explicativas, experimentales y cronológicas, lo que lleva a la continua revisión de métodos de trabajo y esquemas conceptuales. 
Tabla I - Distribución temática de aportaciones a las reuniones nacionales de la Sociedad Española de Geomorfología.

Quadro I - Temas das comunicações apresentadas nas reuniões nacionais da Sociedade Espanhola de Geomorfologia.

Table I - Main research themes in the national conferences of the Spanish Society of Geomorphology.

\begin{tabular}{|c|c|c|c|c|c|c|c|c|c|c|}
\hline Bloque temático & $\begin{array}{r}\text { Reunión I. }^{\mathbf{a}} \\
1990 \\
\end{array}$ & $\begin{array}{r}\text { II. }^{\mathbf{a}} \\
1992 \\
\end{array}$ & $\begin{array}{r}\text { III. }^{\mathbf{a}} \\
1994 \\
\end{array}$ & $\begin{array}{r}\text { IV. }^{\mathbf{a}} \\
1996 \\
\end{array}$ & $\begin{array}{r}V^{\mathbf{a}^{\mathbf{a}}} \\
1998 \\
\end{array}$ & $\begin{array}{r}V^{\mathbf{I I}^{\mathbf{a}}}{ }^{\mathbf{2}} \\
2000\end{array}$ & \multicolumn{2}{|c|}{$\begin{array}{l}\text { VII. }^{\mathbf{a}}{ }^{\text {VIII. }}{ }^{\mathbf{a}} \\
20022004\end{array}$} & Total & $\%$ \\
\hline Morfolodia regional & 3 & - & 3 & - & - & 3 & - & 2 & 11 & 2,1 \\
\hline $\begin{array}{l}\text { Morfología estructural } \\
\text { y neotectónica }\end{array}$ & 6 & 2 & 9 & 4 & 3 & - & - & 3 & 27 & 4,9 \\
\hline Morfología cárstica & 7 & - & 5 & - & 3 & 1 & - & - & 16 & 2,9 \\
\hline Morfología volcánica & - & - & - & - & - & - & 3 & 2 & 5 & 0,9 \\
\hline \multicolumn{11}{|l|}{ Morfología de países frios } \\
\hline Glaciar & 6 & 4 & 4 & 2 & 5 & 4 & 6 & 1 & 32 & 5,9 \\
\hline Periglaciar/crionival & 1 & 1 & 3 & 2 & 1 & 3 & 5 & 2 & 18 & 3,4 \\
\hline Morfología de zonas áridas & 8 & - & 3 & - & - & - & - & - & 11 & 2,1 \\
\hline Morfología litoral & 7 & 5 & 8 & 2 & 7 & 10 & 9 & 7 & 55 & 10,2 \\
\hline Morfología fluvial & 12 & 9 & 9 & 6 & 16 & 15 & 5 & 4 & 76 & 14,2 \\
\hline Morfogénesis de laderas & 4 & 6 & - & 10 & 3 & 4 & - & - & 27 & 4,9 \\
\hline Litología y modelado & - & 8 & - & - & 5 & 5 & 1 & 4 & 18 & 3,4 \\
\hline Erosión de suelos & 12 & 19 & 22 & 13 & 8 & 5 & 6 & 1 & 86 & 15,9 \\
\hline Geomorfología y suelos & 5 & 6 & 6 & - & - & 11 & 4 & - & 32 & 5,9 \\
\hline Paleoambientes cuaternarios & - & - & - & 6 & 7 & 5 & 2 & 3 & 23 & 4,3 \\
\hline Análisis espacial, cartografía, SIG & - & 5 & 1 & 4 & 2 & - & 3 & 1 & 16 & 2,9 \\
\hline Geomorfología y riesgos naturales & - & - & - & - & 8 & 3 & 4 & $48^{*}$ & 63 & 11,8 \\
\hline Otros temas & 3 & 1 & 3 & - & 1 & 1 & 5 & 9 & 23 & 4,3 \\
\hline Total & 74 & 66 & 76 & 49 & 72 & 66 & 52 & 87 & 542 & 100,0 \\
\hline
\end{tabular}

* La VIII. ${ }^{a}$ Reunión (2004) incluyó, además, el tema monográfco Riesgos Naturales y Antrópicos en Geomorfología con una aportación de 48 trabajos de los que 5 hacían referencia a medios fríos (alta montaña)

De tal manera ha sido importante la labor de IPA-España en el ámbito de la Península Ibérica que, en 2005, Portugal, siguiendo el ejemplo español, se ha integrado como miembro asociado en la Internacional Permafrost Association bajo la denominación IPA-Portugal. La colaboración entre las dos comunidades ibéricas culminará en 2007 con la celebración conjunta de sus reuniones nacionales. Ésta tendrá lugar en la serra da Estrela y estará organizada por el grupo de la Universidade de Lisboa.

\section{ANÁLISIS DE LA PRODUCCIÓN CIENTÍFICA MÁS RECIENTE. LAS REUNIONES DE IPA-ESPAÑA.}

El mejor documento para evaluar las publicaciones más recientes sobre morfogénesis periglaciar son las ediciones de las diferentes actas de las reuniones de IPA-España (donde se incluye la aportación portuguesa), que se sintetizan en la tabla 2. Su análisis permite abordar las áreas de estudio, temas preferentes, métodos de trabajo y resultados más significativos y grupos de trabajo más persistentes. 
La investigación en geomorfologia periglaciar en España y Portugal

Tabla II - Trabajos presentados en las diferentes reuniones de IPA-España

Quadro II - Trabalhos apresentados nas várias reuniões do grupo IPA-Espanha

Table II - Research presented in the conferences of the group IPA-Spain

\begin{tabular}{lcccccc}
\hline Reunión(año) & Conferencias & $\begin{array}{c}\text { Trabajos } \\
\text { de campo }\end{array}$ & Comunicaciones & $\begin{array}{c}\text { Ambito } \\
\text { España }\end{array}$ & $\begin{array}{c}\text { Ambito } \\
\text { Portugal }\end{array}$ & $\begin{array}{c}\text { Otros } \\
\text { ámbitos }\end{array}$ \\
\hline I. $^{\text {a }}$ (1994) & 2 & 1 & - & - & - & - \\
II. $^{\text {a }}(1995)$ & - & 2 & - & - & - & - \\
III. $^{\text {a }}(1997)$ & 2 & 2 & 17 & 11 & 1 & 5 \\
IV. $^{\text {a }}(1999)$ & 1 & 1 & 15 & 14 & 1 & - \\
V. $^{\text {a }}{ }^{\text {(2001) }}$ & 1 & 1 & 14 & 9 & 1 & 4 \\
VI. $^{\text {a }}(2003)$ & 2 & 1 & 16 & 9 & 2 & 5 \\
Total & 8 & 8 & 62 & 43 & 5 & 14 \\
\hline
\end{tabular}

\section{1. Áreas de estudio}

Las áreas estudiadas en los 62 trabajos se distribuyen de la siguiente manera: 43 se refieren a España, 5 a Andorra, 5 a Portugal, 1 a las Islas Shetland del Sur (Antártida), 1 a Tarfala (Suecia), 1 al volcán Popocatepétl (México) y 1 a la Península de Varanger (Noruega). Respecto a los que se ocupan de la Península Ibérica (53), la casi totalidad, se centran en espacios de montaña destacando el Pirineo Central (13), Sistema Central (8), sierras de la Estrella (5) y Gredos-Peñalara (3), Béticas (6), Sierra Nevada (5) y Sierra de los Filabres (1), Cordillera Cantábrica (4), Macizos gallegos (3), Sistema Ibérico (2) y Montes de Toledo (1). El resto de trabajos, 3, coinciden en ámbitos extramontañosos: depresión de Vera y rebordes montanos (1), Submeseta sur en su contacto con el ramal meridional de la Cordillera Ibérica (1) y Submeseta sur en su enlace con Sierra Morena (1).

Acerca de las áreas preferentes destacan el Pirineo Central y Sierra Nevada, las montañas más elevadas de la Península Ibérica y en las que es notoria la actual presencia de pisos morfogénicos fríos diferenciados. Por lo que se refiere a las áreas desatendidas hay que incluir a los sistemas montañosos del levante mediterráneo y, en concreto, a los del País Valenciano y extremos más septentrionales de las Béticas, en territorio de Alicante y de Murcia, donde hace ya tiempo se describieron modelados periglaciares pleistocenos, particularmente en lo relativo al tapizado de laderas (Rosselló, 1970; López Bermúdez, 1973; Calvo Cases, 1987) y recientemente confirmados por Marco Molina (2001). Lo mismo podría decirse, aunque en menor medida, del Sistema Costero Catalán, a pesar, también, de la noticia referida al Montseny, donde se identificaron nichos glacionivales y espesos mantos de derrubios ordenados regularizando vertientes (Llobet, 1975/76). Fuera de estos ámbitos también se hecha en falta una particular atención a la Submeseta norte, en concreto al valle del Duero, donde García Fernández (comunicación personal, 1979) ya mostrara depósitos afectados por gelifluxión, y a todo el conjunto del País Vasco y Pirineo Occidental, con morfologías periglaciares heredadas (Ugarte et al., 1984). Con respecto a Portugal se detecta un interés continuado por los modelados de origen frío, en particular en serra da Estrela, por lo que se refiere a dinamica actual y herencia (Vieira, 1998, 2004; Vieira et al. 2003). En las restantes montañas la atención se ha centrado en depósitos de ladera y, en algunos casos, en dinámica actual. Son los casos de las sierras del Minho (Peneda y Geres - Coudé-Gaussen, 1979, 1981; Ferreira et al., 1992, 1999; Vieira, 1995; Marão - Pedrosa, 
1993; Caramulo - Cordeiro, 1993, 2004; Lousã - Daveau, 1973; Lourenço, 1996) o de las sierras del entorno del Macizo Calcário Estremenho (Cunha, 1990; Rodrigues, 1988, 1998). En semejantes términos también habría que contemplar a las islas Baleares, Canarias y Madeira en las que, igualmente, se describieron modelados de procesos fríos (Rosselló, 1977; Höllermann, 1978; Ferreira, 1981; etc.), incluso actuales (Morales et al., 1977; Ferreira, 1981).

\section{Temas preferentes}

Por lo que respecta a la temática dominante existe una gran diversidad de intereses que podrían agruparse en dos tendencias: estudio de formas heredadas y estudio de formas actuales. Una y otra tendencia, a su vez, tiende a afrontarse desde una perspectiva general y desde otra particular. La primera, por ejemplo, supone descripciones cualitativas de formas de modelado y su distribución clasificada en altura, lo que permite ensayar una zonación de pisos morfodinámicos. Esto es lo que ocurre cuando de un sector bien delimitado se obtiene una síntesis producto de anteriores trabajos parciales, como ha venido sucediendo para determinadas unidades del Pirineo Central, conjunto de Galicia, Sierra Nevada, Islas Shetland del Sur y Norte de Portugal.

Otros trabajos son de carácter más específico y se basan en el análisis de formas de modelado a partir del estudio de los procesos desencadenantes heredados o actuales. En tal sentido y sobre morfologías heredadas destacan glaciares rocosos (montes de León - Sierra de Gistredo), campos de bloques (montañas de Galicia - Xistral, Ancares, Courel, Trevinca, etc.) y, particularmente, derrubios de ladera (tipo estratificado y/o asistido), que son los depósitos más extendidos en nuestras montañas. Este último tema ha ocupado atención preferente en el Pirineo Central (eje axial y Prepirineo) y Cordillera Ibérica, ramal castellano donde se han determinado diferentes generaciones, algunas datadas a partir de ${ }^{14} \mathrm{C}$, radiometría y artefactos prehistóricos. Igualmente han ocupado interés destacado en Sierra de Filabres, en el tránsito Meseta Sur y Sierra Morena oriental y en los llanos de Vera-Sorbas (Almería), donde los coluvios periglaciares podrían haber quedado establecidos por encima de los 700-1 000m.

En cuanto a procesos fríos actuales y formas desencadenantes las aportaciones resultan numerosas, aunque focalizadas en tramos de montañas donde las condiciones climáticas son propicias. Así ocurre, por ejemplo, con el comportamiento térmico del aire (Andorra), o con aquellos otros trabajos referidos al estudio de la evolución térmica de la capa activa, a partir de la monitorización de puntos significativos (Pirineo Oriental - macizo de Calmquerdós -, Sierra Nevada, Base Antártica Española, Centro de Portugal - serra da Estrela, etc.), lo que ha implicado el diseño particular de protocolos de actuación in situ. También el régimen de temperaturas ocupa lugar relevante en la clasificación y distribución altitudinal de la morfodinámica, como sucede en la propuesta hecha para Picos de Europa.

En otras ocasiones los trabajos se interesan por el origen y génesis de determinadas microformas a partir del seguimiento monitorizado de procesos (crioturbación, gelisolifluxión, crio-reptación), como ocurre con las figuras geométricas y el desplazamiento de coladas de piedras en Sierra Nevada. En semejante línea de actuación, aunque desde perspectivas más integradoras, destacan las experiencias que se vienen asumiendo acerca del cometido de la nieve en la morfodinámica de Dos Hermanas-Peñalara y en el circo de Gredos (Sistema Central). Igualmente la monitorización ocupa atención particular en el control del movimiento de glaciares rocosos. Los resultados que 
se vienen acumulando en Las Argüalas (Valle del Tena, Pirineo aragonés) y en el Corral del Veleta (Sierra Nevada) están demostrando la bondad de la fotogrametría digital y del GPS como herramientas valiosas. También la fotogrametría está siendo utilizada para la prevención de lahares en el volcán de Popocatepétl (México), a partir del retroceso de la masa glaciar del referido volcán. El seguimiento de la superficie de los glaciares pirenaicos es otra de las preocupaciones que viene atendiéndose desde 1985, al amparo del Programa ERHIN. Los resultados hasta finales del siglo XX, comparados con los que se ofrecieron para 1980 (Serrat, 1981) muestran merma continuada de masa helada. Relacionado con esta temática hay que resaltar, también, los trabajos dedicados a la localización de permafrost alpino en el macizo de Posets (Pirineo Central), donde se ha detectado por encima de los $2700 \mathrm{~m}$. Igualmente se ha localizado en Picos de Europa, en Jou Negro. Y recientemente, también, en el Corral del Veleta (Sierra Nevada), a $3150 \mathrm{~m}$ de altura. En este caso, además, se estudia su relación con las formas asociadas, como igualmente se hace en las Islas de Shetland del Sur.

En otro orden de cosas y referido a acontecimientos históricos de áreas glaciadas debe subrayarse la reconstrucción ambiental a partir del siglo XV, en concreto de la Pequeña Edad del Hielo. En tal sentido sobresalen los estudios que se llevan a cabo en el sur de Andorra, en el valle del Madriu, donde se han detectado diferentes pulsaciones climáticas frías (entre los siglos XV y XIX) a partir del estudio liquenométrico en morrenas de nevero. Y por lo que respecta a los tiempos más recientes, siglos XIX y $\mathrm{XX}$, las reconstrucciones del Corral del Veleta y Maladeta, aunque ahora tomando como referencia documentos de época.

Hay que resaltar en esta relación de temas de estudio la escasez de trabajos referidos a aludes de nieve, pues sólo en una ocasión esta cuestión se trata (valle de Ordesa). Igual podría decirse acerca de la dinámica del paisaje supraforestal o de sus ecosistemas, particularmente contemplando al hombre como agente geomórfico directo o inductor. Sobre estas cuestiones sólo dos trabajos se han contabilizado. Uno, referido al deterioro de formas periglaciares como respuesta a la apertura de pistas de esquí en la estación de Valgrande-Pajares. Otro, preocupado por la incidencia del ganado trashumante en la evolución de formas de modelado de ladera en el macizo de Casamanya (Andorra), particularmente desde que la cabaña ganadera ha tendido a reducirse.

\section{Métodos y técnicas}

Acerca de métodos y técnicas de trabajo empleados igualmente debe destacarse que resultan variados. Sin lugar a dudas el análisis in situ que proporciona el trabajo sistemático de campo continúa acaparando la mayor atención. Es él, junto a la cartografía geomorfológica de detalle $(1 / 5000,1 / 10000,1 / 25000)$ elaborada en una primera fase a partir de fotointerpretación, quien sustenta los trabajos de reconocimiento de formas de modelado.

El conocimiento del comportamiento climático en nuestras montañas se desconoce con cierta precisión por lo que supone un grave inconveniente a la hora de establecer pisos o secuencias morfogénicas altitudinales. Esta situación tiende a mitigarse a partir del tratamiento estadístico y la proyección en altura de los datos de estaciones del llano o enclavadas a media ladera. Los resultados, siempre que estén avalados convenientemente por conjuntos de formas de modelado resultantes y, en su caso, por el tipo de colonización vegetal, permiten tener una aproximación cualitativa del reparto y escalonamiento de procesos fríos en el conjunto de la montaña. La instalación reciente 
de estaciones automáticas completas y sensores autónomos y automatizados de larga duración en parcelas experimentales ha supuesto un gran avance, sobre todo, en el conocimiento del ritmo térmico en superficie y en la capa activa del suelo, a diferentes profundidades. Las experiencias iniciadas en la Antártida, en 1995, y proseguidas y mejoradas en varios enclaves de las montañas de la Península Ibérica (Pirineo, Sierra Nevada, Gredos, Estrela) están resultando de gran interés, pues los resultados que vienen acumulándose permiten explicar mejor el comportamiento mecánico de los suelos y, en particular, lo relativo a procesos y formas instalados en la capa activa.

La morfometría y la sedimentología continúan siendo técnicas muy empleadas en el análisis de los derrubios de ladera (groize, grèze), en las coladas de bloques y en los debris flows, particularmente para determinar secuencias deposicionales y génesis de los arrastres, tal como se detecta en los trabajos realizados sobre grèzes y groizes litées en el Pirineo Central - núcleo axial y Prepirineo -, Sierra de Albarracín, Cordillera Ibérica-rama castellana, Galicia oriental y Sierra de Filabres. Y por lo que respecta a coladas y debris flows, las experiencias de Tarfala (Suecia) resultan significativas. En la mayoría de los casos y determinadas las condiciones de formación de los depósitos, éstos, además, han sido datados a partir de ${ }^{14} \mathrm{C}, \mathrm{U} / \mathrm{Th}$, artefactos arqueológicos, etc., lo que ha permitido situarlos en el tiempo, la mayoría entre el Pleistoceno medio y Subboreal $y$, en ocasiones y gracias a la Palinología, determinar los paleoambientes en los que se construyeron, como sucede en los casos de Bentué de Ransal y el Alto valle del Cinca (Pirineo Central).

En otras ocasiones determinadas técnicas de datación han permitido acercarnos más a nosotros y determinar etapas frías históricas dentro de la Pequeña Edad del Hielo, por ejemplo. Así ha sucedido con la liquenometría, ya comentada para el caso del sur de Andorra. En este mismo rango cronológico, aunque desde diferente perspectiva, la documentación histórica de época está dando resultados excelentes en la reconstrucción del paisaje altimontano de las montañas glaciadas. En tal sentido, los escritos de viajeros de la Ilustración y posteriores de naturalistas se están convirtiendo, cada vez más, en referencias valiosas, particularmente si relatan montañas en las que pudo haber actuado la Pequeña Edad del Hielo. Al respecto, el bagaje de información obtenido de Sierra Nevada y Pirineo central resulta significativo.

Igualmente se está poniendo de relieve la utilidad que poseen los mapas de cobertura nival en la explicación de los procesos y formas asociadas a la nieve a gran escala espacial. La realización sistemática y periódica de fotografías de cobertura nival y su conversión digitalizada a mapas, convierte a éstos en herramientas muy eficaces a la hora de interpretar el reparto espacial de determinados procesos morfogénicos y modelados asociados. Las experiencias que se llevan a término en Dos Hermanas-Peñalara (Sistema Central), inmersas en una filosofía geográfica integradora resultan novedosas y de gran interés. En similar línea habría también que citar los ensayos acerca de las relaciones entre especies vegetales y pedreras de alta montaña (Sierra de Gredos), aspecto geomorfobiológico que ya fue ensayado en Pirineo oriental (Soutadé, 1978; Serve, 1989), desde la perspectiva geomorfológico y geobotánica, respectivamente.

Las técnicas geofísicas igualmente han estado presentes en determinados trabajos, en particular en aquéllos dedicados a la detección de permafrost profundo y a su estado físico, pues su empleo ha permitido localizar el reparto extensivo y potencia de estas masas heladas en diferentes enclaves montañosos. Las más fecundas experiencias se han centrado en Posets (Pirineo Central), donde en relación a la altitud del macizo se ha distinguido permafrost esporádico, discontínuo y contínuo. Experiencias recien- 
tes también se han llevado a cabo en Sierra Nevada. En ambos casos los resultados obtenidos han sido fruto de diferentes protocolos de actuación (refracción sísmica, SEV, geo-radar, etc.), completados con mediciones de BTS.

El control de la movilidad de los glaciares rocosos, a partir de puntos fijos instalados en diferentes lugares de su superficie, ha supuesto el empleo de técnicas topográficas, geodésicas y fotogramétricas. Los resultados obtenidos, de gran interés, validan la fiabilidad de ellas. En tal sentido, sobresalen los trabajos llevados a cabo en Las Argüalas (Pirineo Central), desde 1991 hasta 2000. También los iniciados en el Corral del Veleta (Sierra Nevada) en el verano del 2000. La bondad de estas técnicas abre expectativas en el control de la inestabilidad de determinadas laderas, en particular las afectadas por flujos y deslizamientos gelifluidales y/o soligelifluidales.

\section{Perfil y procedencia de los autores}

Acerca del perfil y procedencia de los autores, siempre instalados en universidades o centros de investigación, hay que reseñar que la casi totalidad son geomorfólogos cuaternaristas y dentro de ellos predominan los geógrafos, seguidos de geólogos. También es relevante la presencia de físicos y edafólogos y, recientemente, la de cartógrafos y geodestas. Y en cuanto a la organización de su labor reseñar la predominancia del trabajo en equipo, caracterizado, éste, por la pluridisciplinariedad.

En cuanto a la autoría de los trabajos resalta el escaso número de aportaciones firmadas por un solo autor. De los 62 registros que venimos comentando esto sólo se cumple en siete ocasiones (2, en 1997; 1, en 1999; 1, en 2001; 3, en 2003), pues el resto corresponde a grupos, lo que viene a mostrar que la producción científica se afronta desde equipos de trabajo movidos por intereses científicos comunes y que, por lo general, centran atención preferente en determinados ámbitos geográficos y temáticas (tabla 3 y figura 1). También señalar de estos 62 trabajos que en 50 ocasiones todos los firmantes son españoles. La participación extranjera se cumple en 12 casos (Portugal, Francia, Reino Unido, USA, Noruega, México) y de ellos, en 4 títulos, resulta total. En cuanto a la procedencia de las firmas españolas existe una predominancia de Departamentos Universitarios (Geografía, Geología, Física, Edafología, Ingeniería del Terreno, Ingeniería Geológica, Medio Ambiente, Fotogrametría, etc.), seguida de unidades de investigación del CSIC y de diferentes organismos de la Administración pública. Muy puntualmente aparecen empresas privadas.

\section{CONCLUSIONES. HACIA NUEVAS PERSPECTIVAS}

El interés por los temas periglaciares entre los geomorfólogos y geógrafos físicos españoles y portugueses durante estas últimas décadas ha sido notable y se ha venido constatando en la producción científica publicada y en la calidad de las aportaciones, en las que se detecta una constante renovación de ideas y métodos de trabajo, como se ha reflejado recientemente en el número 30 (2004) de la revista Cuadernos de Investigación Geográfica, monográfico dedicado a procesos geomorfológicos en medios fríos. También habría que subrayar en este devenir reciente que la labor española y portuguesa ha empezado a valorarse positivamente entre la comunidad internacional, sobre todo europea, y muy en especial a partir del proyecto PACE (Permafrost and Climate in Europe) y más recientemente a raíz de la creación de la red European Science Foundation-PACE 
21, en los que España y Portugal han venido participando. Como también lo ha venido haciendo hecho en la I. ${ }^{a}$ Conferencia Europea del Permafrost (Roma, 2001), la VIII. ${ }^{a}$ Conferencia Internacional del Permafrost (Zurique, 2003) y la II. ${ }^{a}$ Conferencia Europea del Permafrost (Potsdam, 2005).

En su conjunto, los resultados científicos acumulados a lo largo de esta última década han venido a demostrar la existencia de una dinámica geomórfica fría generalizada con repercusión en las formas de modelado en el conjunto de la Península Ibérica, aunque con mayor intensidad en las montañas, lo que atestigua que el paisaje reciente de ellas, el morfológico, particularmente, está muy asociado a sistemas de procesos de erosión vinculados a la acción combinada del hielo, frío y nieve a lo largo del Pleistoceno. Y por lo que respecta al actual dominio de estos sistemas de procesos se ha demos-

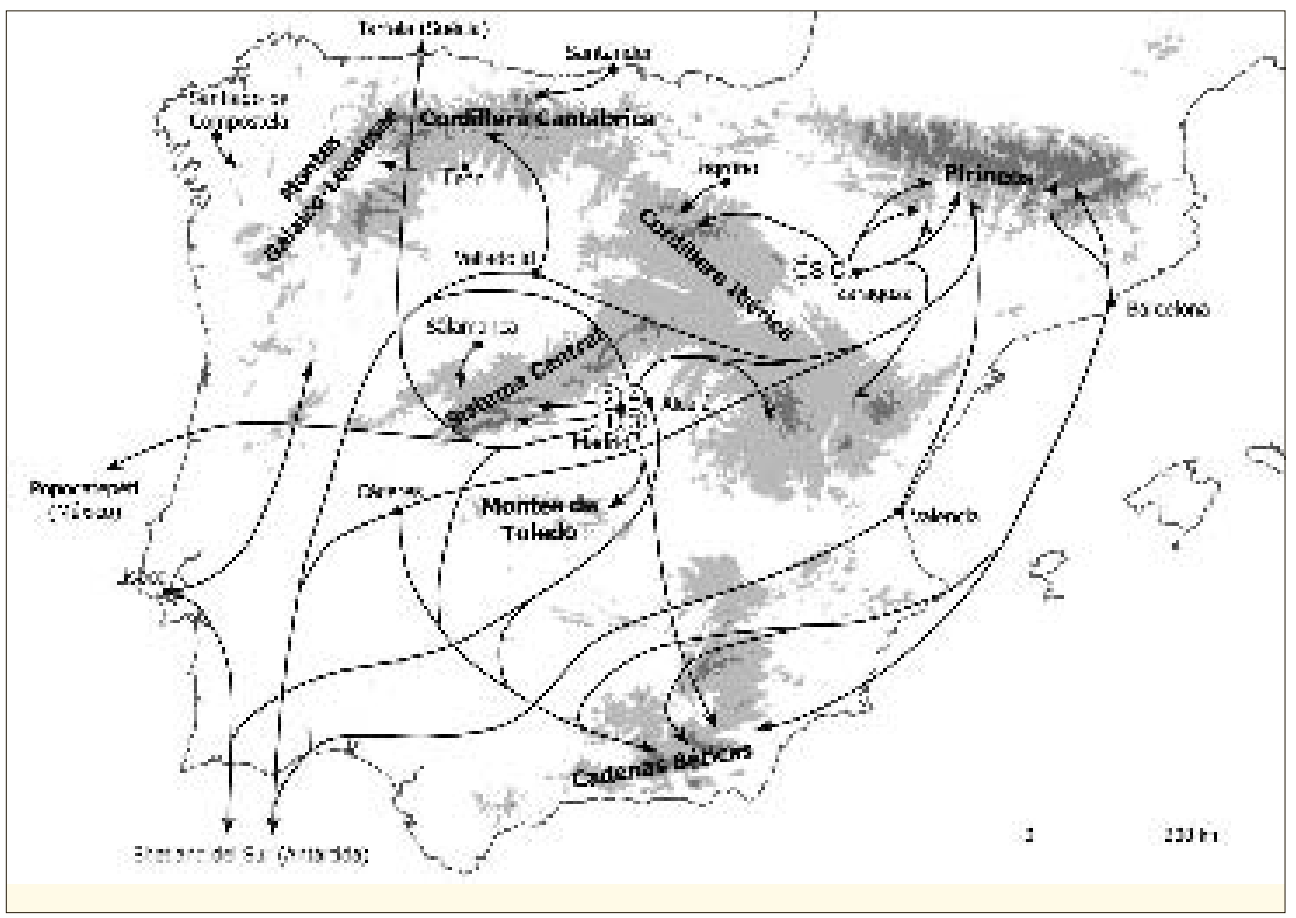

Fig. 1. Grupos de investigación y áreas preferentes de estudio.

1-3 - Grupos de la Universidad Complutense de Madrid, 4 - Grupo de la Universidad Autónoma de Madrid (detalles en la tabla 3)

Fig. 1 - Grupos de trabalho e principais regiões de estudo.

1-3 - Grupos da Universidade Complutense de Madrid,

4 - Grupo da Universidade Autónoma de Madrid (ver pormenores no quadro 3)

Fig. 1 - Working groups and main research regions.

1-3 - Groups of the Complutense University of Madrid,

4 - Group of the Autonomous University of Madrid

(see details in table 3) 
Tabla III - Grupos de trabajo consolidados, áreas geográficas y tendencias temáticas preferentes

Quadro III - Grupos de trabalho, regiões e temáticas de investigação

Table III - Working groups, regions and themes of research

\begin{tabular}{|c|c|c|}
\hline Grupo de trabajo & Área geográfica preferente & $\begin{array}{l}\text { Predominancia de temas } \\
\text { tratados }\end{array}$ \\
\hline Grupo de Zaragoza & $\begin{array}{l}\text { Pirineo Central, Prepirineo } \\
\text { Central, Sistema Ibérico } \\
\text { (ramal sur). }\end{array}$ & $\begin{array}{l}\text { Derrubios estratificados. } \\
\text { Catalogación de procesos } \\
\text { y formas. Cronología. }\end{array}$ \\
\hline Grupo del CSIC (Jaca-Zaragoza) & $\begin{array}{l}\text { Pirineo Central, Prepirineo } \\
\text { Central, Sistema Central } \\
\text { (ramal norte). }\end{array}$ & $\begin{array}{l}\text { Derrubios estratificados. } \\
\text { Zonación de procesos y formas. } \\
\text { Cronología. }\end{array}$ \\
\hline Grupo de Madrid (Complutense 1) & $\begin{array}{l}\text { Sistema Central, Tarfala } \\
\text { (Suecia). Popocatepétl } \\
\text { (México). }\end{array}$ & $\begin{array}{l}\text { Morfodinámica nival. Debris flows. } \\
\text { Prevención de lahares. }\end{array}$ \\
\hline Grupo de Madrid (Complutense 2) & Sistema Central. & Catalogación de formas. Cronología. \\
\hline Grupo de Madrid (Complutense 3) & $\begin{array}{l}\text { Sierra de los Filabres, } \\
\text { Montes de Toledo-Meseta sur. }\end{array}$ & Derrubios de ladera. \\
\hline Grupo de Madrid (Autónoma) & $\begin{array}{l}\text { Cordillera Ibérica ramal } \\
\text { castellano. }\end{array}$ & Derrubios de ladera. Cronología. \\
\hline Grupo de Santiago de Compostela & Macizos gallegos. & $\begin{array}{l}\text { Catalogación de formas } \\
\text { y zonación altitudinal. Cronología. }\end{array}$ \\
\hline Grupo de Barcelona & $\begin{array}{l}\text { Sierra Nevada, Pirineo } \\
\text { Oriental, Andorra, SE. } \\
\text { Península Ibérica. }\end{array}$ & $\begin{array}{l}\text { Monitorización de procesos. } \\
\text { Zonación altitudinal. Glaciarismo } \\
\text { histórico. Evolución de los } \\
\text { estudios periglaciares. }\end{array}$ \\
\hline Grupo de Valladolid & $\begin{array}{l}\text { Pirineo Central, } \\
\text { Cordillera Cantábrica. }\end{array}$ & $\begin{array}{l}\text { Catalogación de formas. } \\
\text { Zonación altitudinal. } \\
\text { Glaciarismo histórico. }\end{array}$ \\
\hline Grupo de Alcalá de Henares-Lisboa & Shetland del Sur (Antártida). & Termicidad capa activa. \\
\hline Grupo de Cantabria & Picos de Europa. & Termometría y modelados. \\
\hline Grupo de León & Montes de León. & Glaciares rocosos heredados. \\
\hline Grupo de Lisboa & $\begin{array}{l}\text { Estrela, Gerês, Macizo } \\
\text { Calcário Estermenho, } \\
\text { Shetland del Sur (Antártida). }\end{array}$ & $\begin{array}{l}\text { Catalogación de formas. } \\
\text { Termicidad del suelo. } \\
\text { Detección remota. }\end{array}$ \\
\hline Grupo de Lisboa-Coimbra & Portugal. & $\begin{array}{l}\text { Historia del periglaciarismo } \\
\text { en Portugal. }\end{array}$ \\
\hline $\begin{array}{l}\text { Grupo de Barcelona-Madrid } \\
\text { (Complutense I) } \\
\text {-Alcalá de Henares-Cáceres }\end{array}$ & Sierra Nevada. & $\begin{array}{l}\text { Permafrost y formas asociadas. } \\
\text { Control glaciar rocoso. } \\
\text { Termicidad del suelo. } \\
\text { Morfodinámica nival. }\end{array}$ \\
\hline $\begin{array}{l}\text { Grupo de Valladolid-Madrid } \\
\text { Autónoma-Cáceres-Valencia }\end{array}$ & $\begin{array}{l}\text { Pirineo Central, } \\
\text { Shetland del Sur (Antártida). }\end{array}$ & $\begin{array}{l}\text { Permafrost. Catalogación de formas. } \\
\text { Control glaciares rocosos. Aludes. }\end{array}$ \\
\hline
\end{tabular}


trado su eficacia en los niveles supraforestales (superada el timberline), muy visibles en el Pirineo y Sierra Nevada, que son los sistemas montañosos con niveles de cumbres que superan los $3000 \mathrm{~m}$.

El camino recorrido ha sido importante como así también los logros. Ahora, y a la vista de lo conseguido, lo que cabe es reflexionar y preguntarse hacia donde deben encaminarse las investigaciones. En tal sentido, parece oportuno, a la vista de los últimos trabajos aparecidos, señalar el interés que vienen mostrando diferentes programas de actuación. Entre ellos destacan:

A. Estudio de la génesis y determinación cronológica de formas de modelado heredadas y subactuales.

De entre los temas preferentes por su significado paleoclimático destacan los derrubios de ladera (tipo grèze litée y groize) y los glaciares rocosos. Sobre los primeros interesa resaltar que son testigos morfológicos muy fiables en la determinación de los límites inferiores de los procesos fríos, adquierendo mayor significado cuando sus lechos pueden ser datados $\left({ }^{14} \mathrm{C}\right.$, U/Th, artefactos prehistóricos, fósiles, etc.), como viene ocurriendo en las Cordilleras Béticas, Sistema Ibérico y Prepirineo central. Y por lo que respecta a glaciares rocosos destacar, igualmente, que su progresivo descubrimiento en montañas débilmente glaciadas, ramal castellano de la Cordillera Cantábrica - por ejemplo, viene a ratificar la amplitud espacial de regímenes periglaciares en la Península Ibérica durante el Pleistoceno-holoceno.

B. Reconstrucción ambiental holocena del paisaje de la alta montaña.

Se trata de una perspectiva geográfica que tiende a explicar la evolución del paisaje en los medios fríos de las montañas desde interpretaciones globales. En ello, no solamente los modelados de relieve poseen un significado particular, por su configuración topográfica y fijación espacial-altitudinal, sino también todos aquellos otros datos asociados a la génesis del depósito, como los análisis morfométricos, sedimentológicos y morfoedáficos. También resulta de gran interés la información de carácter paleobiogeográfico, particularmente cuando se toman como referencia y analizan polínicamente con datación absoluta perfiles de turberas y lóbulos de gelifluxión vegetalizados. La combinación de resultados y su interpretación global está llamada a ser una sólida aportación en la reconstrucción ambiental holocena del paisaje de la alta montaña.

\section{Estudio de la deglaciación histórica de la Pequeña Edad del Hielo.}

Entre el periodo de los siglos XV-XIX, en la denominada Pequeña Edad del Hielo, el incremento de los glaciares alpinos tuvo su reflejo en determinadas montañas de la Península Ibérica. El estudio de la deglaciación y de sus etapas de estos enclaves es uno de los temas a los que se vienen dedicando esfuerzos, sobre todo en Pirineo central y oriental, Sierra Nevada y Picos de Europa. Y viene haciéndose desde diferentes perspectivas:

a) Análisis de formas de modelado;

b) Documentación histórica (a partir del siglo XVIII, sobre todo) y

c) Análisis liquenométricos de depósitos.

D. Monitorización de procesos morfogénicos fríos actuales. Dinámica del paisaje.

Este bloque temático incide en el estudio de los procesos fríos actuales y su relación con la dinámica del paisaje. En la actualidad los esfuerzos se centran en el control 
de la estabilidad de la fracción mineral a partir del seguimiento térmico del permafrost alpino y de su estado físico y del manto nival y formas de modelado asociadas. Los enclaves montañosos donde ya se trabaja en tal sentido se sitúan en Gredos, Estrela, MaladetaPosets, Sierra Nevada. Asimismo se están sentando las bases para la creación de una red de seguimiento climático en medios supraforestales de las montañas de la Península Ibérica. Su finalidad es determinar las relaciones que existen entre manto nival - temperatura de la capa activa, estabilidad del suelo y sus posibles conexiones con el denominado cambio global.

\section{E. Riesgos naturales y cartografía de riesgos.}

Es la vertiente aplicada más cultivada en los estudios de medios fríos en la Península Ibérica. Trabajos recientes han sido llevados a término en el Pirineo oriental y Principado de Andorra. Éstos vienen focalizando atención en la detección de inestabilidad de laderas, particularmente, en los casos de derrumbes de rocas y deslizamientos. También existen experiencias en la determinación de aludes de nieve (Furdada, 1996). Tanto en un caso como en otro los objetivos tienden a analizar parámetros desencadenantes y ambientes favorables al desarrollo de tales eventos y, a partir de ellos, determinar probabilidades de acontecimientos y grado de peligrosidad. En este último sentido, la elaboración de mapas de susceptibilidad de riesgos ocupa lugar destacado.

\section{F. Suministro de datos base para la planificación de usos del suelo}

Esta línea de actuación resulta igualmente geográfica, pues lo que se pretende ahora es analizar y diagnosticar el estado real y potencialidad de los sistemas naturales de nuestras montañas con la finalidad de suministrar datos significativos para su mejor gestión. En el caso de los niveles supraforestales acerca de su inestabilidad ante determinadas actuaciones, por ejemplo, las repercusiones que supone la alteración y reducción de pastizales por la instalación de estaciones de invierno (infraestructuras y pistas de esquí). También y desde perspectivas próximas existen experiencias que tienden a resaltar valores naturales y culturales del paisaje. De entre ellas resalta la catalogación de enclaves singulares de interés geoturístico, como se hizo en la serra da Estrela. O como aquel otro que justificó los valores biofísicos del valle del Madriu (Andorra) para su catalogación como Patrimonio de la Humanidad por la UNESCO.

\section{E. Investigaciones en la Antártida.}

Actualmente los trabajos en temas periglaciares de los investigadores españoles y portugueses en la Antártida se centran en el archipiélago de las Islas Shetland del Sur. Dos cuestiones son las más atendidas: cartografía geomorfológica de los espacios deglaciados y monitorización del estado térmico de la capa activa de estos espacios. El interés de ambos temas resulta evidente. Por un lado, contribuye al conocimiento y distribución espacial de los resultados morfogenéticos fríos de altas latitudes en el modelado. Y por otro, aporta nuevos datos de la evolución de las temperaturas en el suelo, de tanto interés en el debate abierto acerca del denominado cambio climático. Ambos trabajos están encuadrados en sendos proyectos internacionales promovidos por la IPA - International Permafrost Association (TSP, Termal State of Permafrost) y SCAR Standing Scientific Group on Geosciences (ANTPAS, Antarctic and sub-Antarctic Permafrost, Periglacial and Soil Environments), respectivamente. 


\section{REFERENCIAS BIBLIOGRÁFICAS}

Asensio Amor I, González Martín J A (1974) Presencia de materiales detríticos ordenados (grèze litées) en el valle del Tajuña (Caravana-Villarejo de Salvanés). Estudios Geológicos, XXX, 69-73.

Barrère P (1952) Evolution mécanique et nivation sur les versants calcaires de la haute montagne pyrénéenne. Pirineos, 24: 201-213.

Brochu M (1969) Existence d'une zone périglaciaire dans la région sommitale du Pic (o Pico) dans l'île du Pic aux Açores. Zeitschrift fur Geomorphologie, N.F., 13(1): 115-118.

Brosche K U (1978) Formas actuales y límites inferiores periglaciares en la Península Ibérica. Estudios Geográficos, 151: 131-161.

Bullón Mata T (1977) Los fenómenos periglaciares de la Mujer Muerta (Sierra de Guadarrama). $V$ Coloquio de Geografía. AGE-Universidad de Granada, Granada: 35-40.

Calvo Cases A (1987) Geomorfología de laderas en las montañas del País Valenciano. Edicions Alfons El Magnànim, Institució Valenciana d'Estudis i Investigació, València.

Carvalho G S (1964) Areias da Gândara (Portugal). Uma formação eólica quaternária. Publ. Museu Lab. Min. Geol. Fac. Ciências do Porto, 82: 7-32.

Carvalho G S (1955) A Gândara (Portugal) e as Landes da Gasconha (França). Memórias e Notícias, 37: 20-36.

Cordeiro A M R (2004) Dinâmica de vertentes em montanhas ocidentais do Portugal Central. Dissertação de Doutoramento em Letras na Área de Geografia. Universidade de Coimbra.

Cordeiro A M R (1993) Dinâmica de vertentes nos Pleniglaciários e Tardiglaciário wurmianos nas Montanhas Ocidentais do Centro-Norte de Portugal. A importância do gelo de segregação. Actas da II Reunião do Quaternário Ibérico, Madrid: 881-888.

Coudé-Gaussen G (1981) Les Serras da Peneda et do Gerês. Étude géomorphologique. Memórias do Centro de Estudos Geográficos, Lisboa, 5: 254.

Coudé-Gaussen G (1979) Les Serras da Peneda et do Gerês (Minho - Portugal), Formes et formations d'origine froide en milieu granitique. Thèse $3 .{ }^{\text {ème }}$ Cycle. Université Paris I, Paris.

Creus J, García Ruiz J M (1977) Observaciones sobre solifluxión en el alto valle del río Gállego. Trabajos sobre Neógeno-Cuaternario, 6: 57-64.

Cunha L (1990) As serras calcárias de Condeixa-Sicó -Alvaiázere. Geografia Física, Coimbra, 1: 329 .

Daveau S (1978) Le périglaciaire d'altitude au Portugal. Colloque sur le périglaciaire d'altitude du domaine méditerranéen et abords. Strasbourg: 63-78.

Daveau S (1973) Quelques exemples d'évolution quaternaire des versants au Portugal. Finisterra - Revista Portuguesa de Geografia, VIII(15): 5- 45.

Dresch J (1937). De la Sierra Nevada au Grand Atlas, formes glaciaires et de nivation. Mélanges de géographie et d'orientalisme offerts à E.F. Gautiers. Tours: 194-212.

Ferreira A B (1981) Manifestações periglaciárias de altitude na ilha da Madeira. Finisterra - Revista Portuguesa de Geografia, XVI(32): 213-229.

Ferreira A B, Rodrigues M L, Vieira G T (2000) Manifestações herdadas e actuais de climas frios em Portugal. In: Procesos y formas periglaciares en la montaña mediterránea. Instituto de Estudios Turolenses. Teruel: 161-190.

Ferreira A B, Vidal Romani J R, Zêzere J L, Rodrigues M L (1999) A glaciação plistocénica da Serra do Gerês. Vestígios geomorfológicos e sedimentológicos. CEG, AGFA, Lisboa, Rel., 37. 
La investigación en geomorfologia periglaciar en España y Portugal

Ferreira A B, Vidal Romani J R, Vilaplana J M, Rodrigues M L, Zêzere J L, Monge C (1992) Formas e depósitos glaciários e periglaciários da Serra do Gerês-Xurés (Portugal, Galiza). Levantamento cartográfico. Cuadernos Laboratório Xeolóxico de Laxe, 17: 121-135.

Floristán A (1953) El modelado periglaciar. Departamento de Geografía Aplicada del CSIC, Zaragoza.

Furdada G (1996) Estudi de les allaus al Pirineu occidental de Catalunya: predicció espacial i aplicacions de la cartografia. Geoforma-Ediciones, Logroño.

García Rosell L, Pezzi M (1977) Análisis de depósitos periglaciares en el sector central de las Cordilleras Béticas (Andalucía). V Coloquio de Geografía. AGE-Universidad de Granada, Granada: 99-107.

García Ruiz J M (1999) La producción científica de la Geomorfología española y su impacto a través de las publicaciones periódicas. CSIC-SEG, Zaragoza.

García Sainz L (1935) Morfología glaciar y periglaciar de la región de la Noguera (cuenca CincaSegre). Boletín de la Sociedad Geográfica Nacional, 54: 64-110.

Ginés A, Mateu J (1977) Fenómenos de clima frío en el Alt Maestrat. Trabajos del Neógeno Cuaternario, 6: 93-103.

Gómez Ortiz A (1980) Estudio geomorfológico del Pirineo catalán: morfogénesis glacial y periglacial de los altos niveles y vertientes merdionales de los macizos de Calmquerdós, Tossa Plana de Llés y Port Negre (Cerdanya-Alt Urgell). Tesis doctoral, Departamento de Geografía, Universidad de Barcelona, Barcelona.

Gómez Ortiz A, Martí Bono C E, Salvador Franch F (2001) Evolución reciente de los estudios de geomorfología glaciar y periglaciar en España (1980-2000). In Gómez Ortiz A, Pérez González A (ed.) Evolución reciente de la Geomorfología española (1980-2000). SEG y Servei de Paisatge de la UB, Geoforma, Logroño: 139-184.

Gómez Ortiz A, Serrat D (1977) Las formaciones geométricas periglaciares de los altos niveles del Pirineo Oriental. V Coloquio de Geografía. AGE-Universidad de Granada, Granada: 75-81.

Guilcher A (1949) Sur les vallons côtiers suspendus des environs de Lisbonne et leurs analogies avec ceux du littoral breton. C. R. Acad. Sc. Paris, 228 : 1512-1514.

Gutiérrez M, Peña Monné J L (1975) Karst y periglaciarismo en la Sierra de Javalambre (provincia de Teruel). Boletín Geológico y Minero, 86:561-572.

Hamelin L E (1958) Matériaux de géomorphologie périglaciaire dans 1'Espagne du Nord. Revue de Géographie des Pyrénées et du Sud-Ouest, XXV: 241-256.

Hempel L (1960) Límites altitudinales geomorfológicos en Sierra Nevada. Estudios Geográficos, 78: 81-93.

Höllermann P (1978) Soil movements in the subtropical mountain environment of Higt Tenerife (Canary Islands). Colloque sur le périglaciaire d'altitude du domaine mediterranéen et abords. Strasbourg: 91-112.

Höllermann P (1967) Zur Verbreitung rezenter Periglacialer Kleinformen in den Pyrenäen und Ostalpen. Gotinga.

López Bermúdez F (1973) La vega alta del Segura. Clima, hidrología y geomorfología. Departamento de Geografía, Universidad de Murcia, Murcia.

Lourenço L (1996) Serras de Xisto do Centro de Portugal. Contribuição para o conhecimento geomorfológico e geo-ecológico. Dissertação de Doutoramento na área de Geografia Física. Universidade de Coimbra

Llobet S (1975-76) Materiales y depósitos periglaciares en el macizo del Montseny. Revista de Geografia, IX: 35-58. 
Marco Molina J A (2001) Derrubios estratificados en la Sierra de Aitana, Alicante (España). Eria, 51: 79-92.

Martí Bono C, González Martín J A (1979) Nota sobre algunos depósitos coluviales del Alto Aragón. III Reunión Nacional del GETC, Zaragoza: 199-207.

Martínez de Pisón E, Muñoz Jiménez J (1972) Observaciones sobre la morfología del Alto Gredos. Estudios Geográficos, 129: 3-103.

Martins A F (1949) Maciço Calcário Estremenho. Contribuição para um estudo de geografia física. Coimbra.

Messerli B (1965) Beiträge zur Geomorphologie der Sierra Nevada (Andalusien). Juris Verlag. Zurich.

Morales A, Martín F, Quirantes F (1977) Formas periglaciares en Las Cañadas del Teide. Aula de Cultura, Santa Cruz de Tenerife.

Pedraza Gilsanz J (1978) Estudio geomorfológico de la zona de enlace entre las Sierras de Gredos y Guadarrama (Sistema Central Español). Tesis doctoral, Facultad de Geología, Universidad Complutense de Madrid, Madrid.

Pedrosa A S (1993) Serra do Marão. Estudo de Geomorfologia. Dissertação de Doutoramento na área de Geografia Física. Universidade do Porto.

Pellicer Corellano F (1980) El periglaciarismo del Moncayo. Geographicalia, 7-8: 3-25.

Peña Monné J L (1998) Los estudios sobre procesos de clima frío en España: balance y perspectivas. In: Procesos biofísicos actuales en medios frios. Publicacions UB. Barcelona: 43-54.

Peña Monné J L (1980) Estudio geomorfológico de la Conca de Tremp y las sierras prepirenaicas leridanas entre los ríos Segre y Noguera Ribagorzana. Tesis doctoral. Departamento de Geografía, Universidad de Zaragoza, Zaragoza.

Pérez Alberti A (1979) Nuevas observaciones sobre glaciarismo y periglaciarismo en el NO. de la Península Ibérica. La Galicia sudoriental. Acta Geológica Hispánica. Homenatge a L. Solé Sabarís, Barcelona: 441-444.

Pissart A (1999) The UGI Periglacial Comission and its role from 1949 until 1980. Biuletyn Peryglacjalny, 38: 7-12.

Rebelo F (1975) Serras de Valongo. Estudo de Geomorfologia. Biblos, Coimbra, 9: 194.

Rodrigues M L (1998) Evolução geomorfológica quaternária e dinâmica actual. Aplicações ao ordenamento do território. Exemplos no Maciço Calcário Estremenho. Dissertação de Doutoramento em Geografia Física, Lisboa.

Rodrigues M L (1988) As depressões de Minde e de Alvados. Depósitos e evolução quaternária das vertentes. Dissertação de Mestrado em Geografia Física e Regional. Universidade de Lisboa, 208 pp.

Rosselló V (1977) El modelado de las áreas glaciales y periglaciales. Ponencia I. V Coloquio de Geógrafos Españoles. AGE-Universidad de Granada, Granada: 25-28.

Rosselló V (1977) Screes periglaciares en la montaña mallorquina. V Coloquio de Geógrafos Españoles. AGE-Universidad de Granada, Granada: 83-92.

Rosselló V (1970) Clima y morfología pleistocena en el litoral mediterráneo español. Papeles del Departamento de Geografía. Secretariado de Publicaciones, Universidad de Murcia: 79-101.

Sanz Herraiz C (1977) Morfología glaciar en la Sierra de Guadarrama. El modelado de las áreas glaciares y periglaciares (Peñalara-Los Pelados). V Coloquio de Geografía. AGE-Universidad de Granada, Granada: 49-55.

Serrat D (1977) Estudio geomorfológico del Pirineo Oriental (Puigmal, Costabona). Tesis doctoral, Facultad de Geología, Universidad de Barcelona, Barcelona. 
Serrat D (coord.) (1981) Catàleg de les glaceres de la Peninsula Ibérica. Muntanya: 435-440 y 498 -503 .

Serve L (1989) Recherches écologiques sur quelques groupements végétaux de l'étage alpin des Pyrénées Orientales. Thèse, Faculté de Sciences, Université de Perpignan.

Solé Sugrañyes L (1973) Nota sobre el límite inferior de los derrubios estratificados de vertiente (grèze litées) en el sector de Sant Llorenç de Morunys (Pirineo Oriental. Provincia de Lérida). Acta Geológica Hispánica, 8 : 167-173.

Soutadé G (1978) Modelé et dynamique actuelle des versants supraforestiers des Pyrénées Orientales. Thèse, Université de Bordeaux, Bordeaux.

Soutadé G, Baudière A (1970) Végétation et modelés des hauts versants septentrionaux de la Sierra Nevada. Annales de Geographie : 436, 709-736.

Tricart J (1966) Quelques aspects des phénomènes périglaciaires quaternaires dans la Péninsule Ibérique. Biuletyn Peryglacjalny, 15: 313-327.

Tricart J, Cailleux A (1967) Le modelé des régions périglaciares. SEDES, Paris.

Tricart J, Cailleux A (1962) Le modelé glaciaire et nival. SEDES, Paris.

Ugarte F, González Martín J A, Alonso F (1984) Acumulaciones detríticas cuaternarias en el valle del río Oñate (Guipúzcoa). Munibe, 36: 65-91.

Vieira G T (2004) Geomorfología dos planaltos e altos vales da Serra da Estrela. Ambientes frios do Plistocénico Superior e dinâmica actual. Dissertação de Doutoramento em Geografia, área Geografia Fisica. Universidade de Lisboa, Lisboa.

Vieira G T (ed.) (1998) Glacial and periglacial Geomorphology of the Serra da Estrela. Guidebook for the field-trip IGU. Commission on Climate Change and Periglacial Environments. CEG and Department of Geography, University of Lisbon, Lisbon.

Vieira G T (1995) Processos morfogenéticos recentes e actuais na Serra do Gerês. Dissertação de Mestrado em Geografia Física e Ambiente. Faculdade de Letras da Universidade de Lisboa, Lisboa.

Vieira G T, Cordeiro A M R (1998) Geomorfología periglaciária em Portugal. Estado do conhecimiento. In: Procesos biofísicos actuales en medios fríos. Estudios recientes. Publicacions de la Universitat de Barcelona, Barcelona: 347-371.

Vieira G T, Mora C, Ramos M (2003) Ground temperature regimes and geomorphological implications in a Mediterranean mountain (Serra da Estrela. Portugal). Geomorphology, 52, 57-72. 